\title{
Superconductivity from Emerging Magnetic Moments
}

\author{
Shintaro Hoshino ${ }^{1,2}$ and Philipp Werner ${ }^{2}$ \\ ${ }^{1}$ Department of Basic Science, The University of Tokyo, Meguro, Tokyo 153-8902, Japan \\ ${ }^{2}$ Department of Physics, University of Fribourg, 1700 Fribourg, Switzerland
}

(Received 15 April 2015; revised manuscript received 17 July 2015; published 8 December 2015)

\begin{abstract}
Multiorbital Hubbard models are shown to exhibit a spatially isotropic spin-triplet superconducting phase, where equal-spin electrons in different local orbitals are paired. This superconducting state is stabilized in the spin-freezing crossover regime, where local moments emerge in the metal phase, and the pairing is substantially assisted by spin anisotropy. The phase diagram features a superconducting dome below a non-Fermi-liquid metallic region and next to a magnetically ordered phase. We suggest that this type of fluctuating-moment-induced superconductivity, which is not originating from fluctuations near a quantum critical point, may be realized in spin-triplet superconductors such as strontium ruthenates and uranium compounds.
\end{abstract}

DOI: 10.1103/PhysRevLett.115.247001

PACS numbers: 74.20.Mn, 74.25.Dw, 74.70.-b

Spin-triplet superconductivity, in the sense of equal-spin pairing, is believed to occur in a number of correlated materials. The best candidate is the layered compound $\mathrm{Sr}_{2} \mathrm{RuO}_{4}$, where the Knight shift remains unchanged across the superconducting phase boundary, in stark contrast to the behavior expected for spin-singlet pairing [1]. In the iron pnictides, where a spin-triplet superconducting phase has been proposed in early theoretical works [2], the experimental evidence points toward spin-singlet pairing, although in LiFeAs a spin-triplet scenario is still being debated $[3,4]$. The uranium-based superconductors are also possible candidates for spin-triplet pairing. In compounds such as $\mathrm{UGe}_{2}$, UCoGe and URhGe, the superconducting state is found near a ferromagnetic phase and the two orders may even coexist [5-8]. For a deeper understanding of unconventional superconductivity in strongly correlated electron systems with multiple active orbitals, it is thus important to clarify the mechanisms which can lead to spin-triplet pairing.

While a $p$-wave symmetry is usually assumed for the pairing state in spin-triplet superconductors, an $s$-wave spin-triplet pairing is also possible by taking into account the orbital degrees of freedom. The mechanism of this unconventional superconductivity can be easily understood [2,9-15]: same-spin electrons tend to occupy the same site due to the Hund coupling, which favors high-spin states. A new insight in this Letter is that the $s$-wave spin-triplet pairing is closely connected to the emergence of local magnetic moments in so-called Hund metals [16]. This class of materials, which includes ruthenates $[17,18]$ and iron pnictides [19-24], exhibits Hund-coupling-induced correlation effects and characteristic non-Fermi-liquid properties. The underlying phenomenon is spin freezing [17]: In a narrowly defined range of fillings and interaction strengths, long-lived magnetic moments appear in the metal phase of multiband systems with Hund coupling [formation of a large composite spin, see right panel of Fig. 1(a)]. In the absence of long-range order, the emerging local moments will be screened at sufficiently low temperature, so that there is no quantum phase transition associated with spin freezing. However, screening large local moments is difficult, and the Fermi-liquid coherence temperature becomes very low $[16,25]$. Hence, as demonstrated here, a spontaneous symmetry breaking preempts the screening of the moments. While deep in the spin-frozen regime the long-lived local moments order magnetically at low temperatures, the emerging and fluctuating local moments in the spin-freezing crossover regime generate spin-triplet pairing. This leads to the formation of a superconducting dome separating the Fermi liquid metal from the magnetically ordered region and results in phase diagrams that closely resemble those of unconventional superconductors.

We consider a three-orbital Hubbard model whose Hamiltonian is given by

$$
\begin{aligned}
\mathcal{H}= & \sum_{\boldsymbol{k} \gamma \sigma}\left(\varepsilon_{\boldsymbol{k}}-\mu\right) c_{\boldsymbol{k} \gamma \sigma}^{\dagger} c_{k \gamma \sigma}+U \sum_{i \gamma} n_{i \gamma \uparrow} n_{i \gamma \downarrow} \\
& +U^{\prime} \sum_{i \sigma, \gamma<\gamma^{\prime}} n_{i \gamma \sigma} n_{i \gamma^{\prime} \bar{\sigma}}+\left(U^{\prime}-J\right) \sum_{i \sigma, \gamma<\gamma^{\prime}} n_{i \gamma \sigma} n_{i \gamma^{\prime} \sigma} \\
& -\alpha J \sum_{i, \gamma<\gamma^{\prime}}\left(c_{i \gamma \uparrow}^{\dagger} c_{i \gamma \downarrow} c_{i \gamma^{\prime} \downarrow}^{\dagger} c_{i \gamma^{\prime} \uparrow}+c_{i \gamma \uparrow}^{\dagger} c_{i \gamma \downarrow}^{\dagger} c_{i \gamma^{\prime} \uparrow} c_{i \gamma^{\prime} \downarrow}+\text { H.c. }\right),
\end{aligned}
$$

where $i$ is the site index, $\gamma=1,2,3$ the orbital index, $\sigma=$ $\uparrow, \downarrow$ the spin index, and $\bar{\sigma}$ represents the complementary $\operatorname{spin}(\uparrow=\downarrow) \cdot \varepsilon_{k}$ is the dispersion of electrons on the lattice, and $\mu$ is the chemical potential. The interaction terms contain the intraorbital $(U)$ and interorbital $\left(U^{\prime}\right)$ Coulomb repulsions, and the Hund coupling $J$. The parameter $\alpha$ controls the anisotropy in spin space; i.e., $\alpha=1$ corresponds to a spin-rotationally invariant system and $\alpha=0$ to the Ising anisotropic case where the interactions are only 
(a)

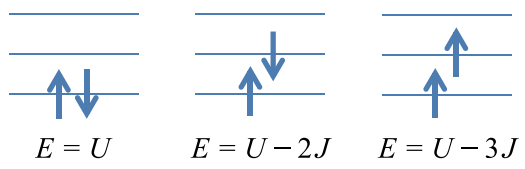

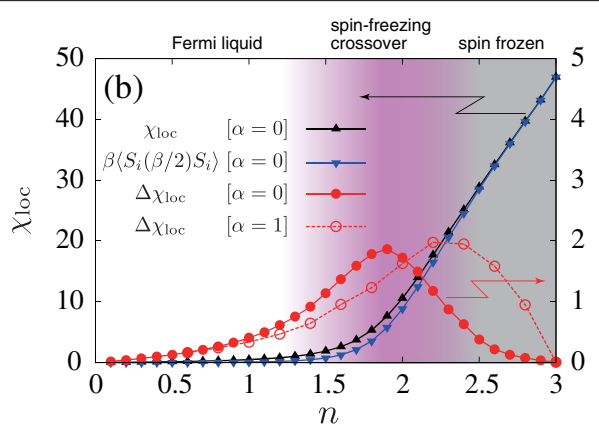

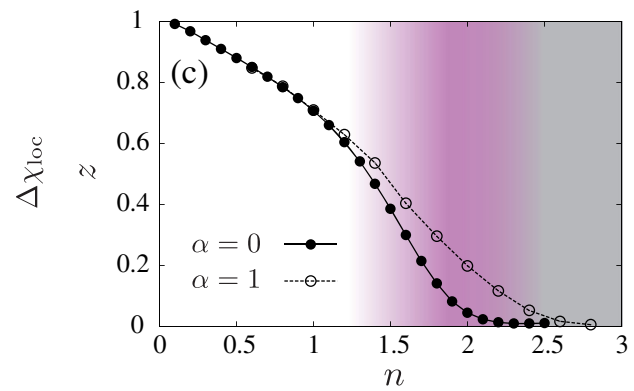

FIG. 1 (color online). (a) Illustration of possible local configurations with two electrons in three orbitals and the corresponding energies $E$. (b) Filling dependence of the local magnetic susceptibilities for $U=1$ and $T=0.005$. The black curve shows the local magnetic susceptibility $\chi_{\text {loc }}$, and the blue curve the contribution from long-lived (frozen) moments. (c) Filling dependence of the renormalization factor $z$ for the same parameters.

of density-density type. A spin anisotropy may originate from spin-orbit coupling, and the parameter $\alpha$ allows us to incorporate this effect in a simple manner [26].

An anisotropic coupling in spin space should, in principle, only change the prefactor of the spin-flip term in Eq. (1). However, for $J>0$ the pair-hopping term, which transfers two electrons in the same orbital to another orbital, is not important. This is because the pair hopping favors the state shown in the left-hand panel of Fig. 1(a), which is hardly realized due to the presence of the intrasite Coulomb interaction $U$, which is larger than $J$. We therefore consider it more convenient to put the anisotropy factor in front of both terms, so that $\alpha$ interpolates between the familiar Ising and rotationally invariant limits.

While the phenomena discussed in this Letter are generic features of multiorbital systems with nonzero Hund-coupling parameter $J$, we will show results for the three-orbital case with $U^{\prime}=U-2 J, J / U=1 / 4$ and consider a semicircular density of states with bandwidth $W=1$. (We neglect specific material effects related to the particular shape of the density of states and to the hybridization between different orbitals, aiming at a general, material unspecific, discussion of the physics.) The model is solved using the dynamical mean field theory [27], combined with a numerically exact continuous-time quantum Monte Carlo method $[28,29]$. This formalism captures local correlation effects.

To illustrate the spin-freezing phenomenon, we compute the dynamic contribution to the local magnetic susceptibility,

$$
\Delta \chi_{\mathrm{loc}}=\int_{0}^{\beta} d \tau\left(\left\langle S_{i}(\tau) S_{i}\right\rangle-\left\langle S_{i}(\beta / 2) S_{i}\right\rangle\right)
$$

where $\beta=1 / T$ is the inverse temperature and $\mathcal{O}(\tau)=$ $\mathrm{e}^{\tau \mathcal{H}} \mathcal{O} \mathrm{e}^{-\tau \mathcal{H}}$. The operator $S_{i}=(1 / 2 M) \sum_{\gamma=1}^{M}\left(c_{i \gamma \uparrow}^{\dagger} c_{i \gamma \uparrow}-\right.$ $\left.c_{i \gamma \downarrow}^{\dagger} c_{i \gamma \downarrow}\right)$, with $M=3$ the number of orbitals, measures the local spin. The first term on the right-hand side yields the local magnetic susceptibility $\left(\chi_{\mathrm{loc}}\right)$. In Eq. (2) we subtract the long-time correlator $\left\langle S_{i}(\beta / 2) S_{i}\right\rangle$, which reflects the magnitude of long-lived frozen moments $[17,30]$. Hence, the quantity $\Delta \chi_{\text {loc }}$ measures the fluctuations of the moments. Figure 1(b) shows the filling dependence of these quantities for $\alpha=0$. While the local susceptibility $\chi_{\text {loc }}$ monotonically increases with increasing $n$, the fluctuation $\Delta \chi_{\text {loc }}$ reaches a maximum at $n \simeq 1.9$. This peak indicates the crossover between the Fermi liquid and spin-frozen regimes, and we use the location of the maximum as our definition of the "spin-freezing crossover." The spin freezing is also reflected in the renormalization factor $z$, or mass-enhancement factor $1 / z$, of the quasiparticles [18]. For the estimation, we use the ansatz $\Sigma(\omega \rightarrow 0)=a+b \omega$ and determine the coefficients by fitting the numerical data. Specifically, we fit the selfenergy by the Padé approximation using the lowest two Matsubara frequencies and compute the renormalization factor by the relation $z=(1-b)^{-1}$. Figure 1(c) exhibits a drop of $z$ in the spin-freezing crossover region. Qualitatively similar results are obtained in the SU(2) symmetric case ( $\alpha=1$, dashed lines).

To study the stability regions of ordered phases, we calculate the susceptibilities

$$
\chi_{\mathcal{O}}=\frac{1}{N} \int_{0}^{\beta}\left\langle\mathcal{O}(\tau) \mathcal{O}^{\dagger}\right\rangle d \tau,
$$

where $N$ is the total number of sites. The operator $\mathcal{O}$ is given by

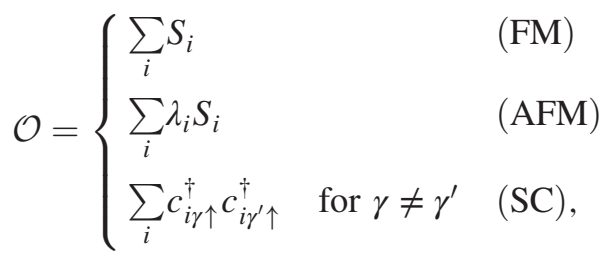

for ferromagnetic order (FM), antiferromagnetic order (AFM), and $s$-wave interorbital spin-triplet superconductivity (SC). $\lambda_{i}$ is a sign that depends on the sublattice. A divergence in $\chi_{\mathcal{O}}$ (or equivalently a sign change in $1 / \chi_{\mathcal{O}}$ ) indicates a possible transition into a long-range ordered 

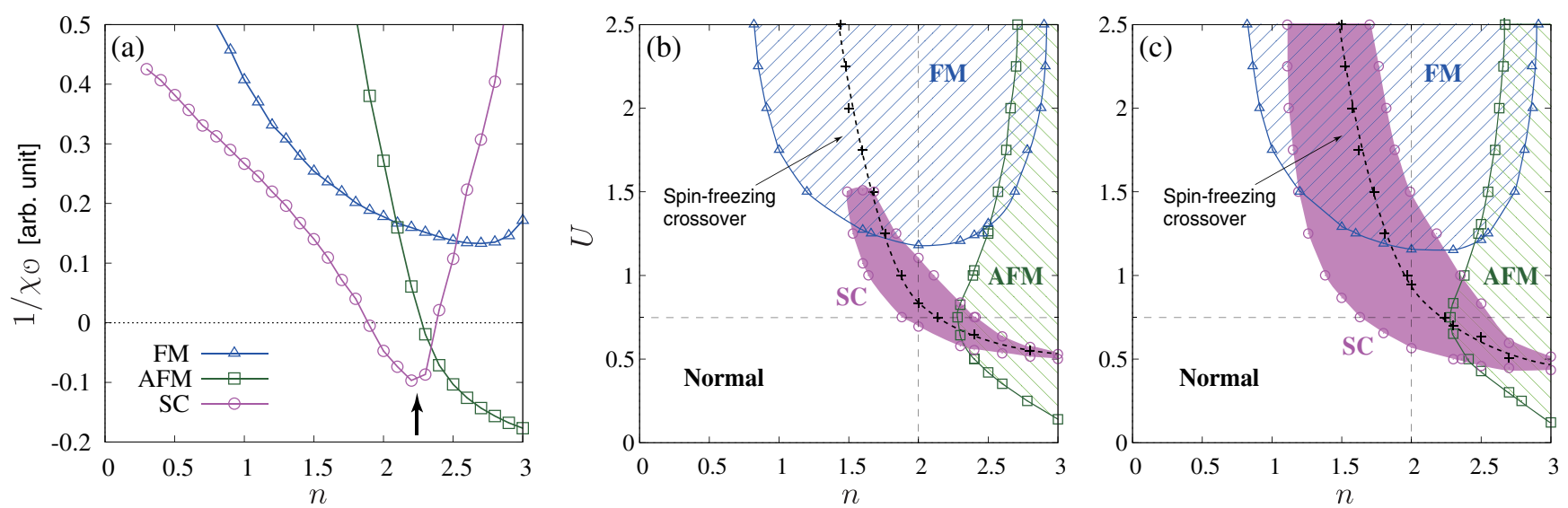

FIG. 2 (color online). (a) Filling dependence of the inverse susceptibilities for FM, AFM, and SC orders for $U=0.75, T=0.005$ in the system with Ising spin anisotropy $(\alpha=0)$. A negative $1 / \chi_{\mathcal{O}}$ indicates a long-range ordered phase with order parameter $\mathcal{O}$. (b), (c) Interaction-filling phase diagrams at $T=0.005$ and $T=0.0025$, respectively. The black dashed line shows the location of the spinfreezing crossover in the system without long-range order.

phase. The susceptibilities in Eq. (4) can be derived from the two-particle Green function [31]. We calculate the vertex part of this Green function from the local impurity problem, and obtain the lattice two-particle Green function by solving the Bethe-Salpeter equation. While we have also calculated the susceptibilities for other types of orders, such as orbital ordering, only the quantities listed in Eq. (4) diverge in the parameter regions considered in this Letter.

Figure 2(a) shows the inverse susceptibilities for $T=0.005, \quad U=0.75, \quad \alpha=0$, and different fillings. Symmetry broken phases exist in the regions where $1 / \chi_{\mathcal{O}}<0$. Repeating this analysis for different $U$, we obtain the $T=0.005$ phase diagram shown in Fig. 2(b). At $U \gtrsim 1.25$, a FM phase appears [32], while near half filling the AFM phase is stable. A new result is the existence of a SC region connecting the FM and AFM phases. This spin-triplet SC phase is clearly associated with the spin-freezing crossover, indicated by the black dashed line, which suggests that the fluctuating local moments at the border of the spin-frozen regime induce the pairing. We also show the phase diagram at a lower $T=0.0025$ in Fig. 2(c), where the SC region expands.

We note that there is no direct attraction among electrons, although the superconductivity is realized by forming electron pairs. The effective attraction for Cooper pairs can be understood from the imbalance of the Coulomb interactions [14,33-35]. To see this, let us consider the situation with two electrons on one lattice site. As shown in Fig. 1(a), there are three kinds of configurations. The energetically most favorable one is the electron pair with the same spins, because of the Hund coupling $J$. Hence, two same-spin electrons will tend to occupy the same site (but different orbitals), even though a repulsive Coulomb interaction acts between them. Indeed, the local effective interaction $\tilde{U}$ among same-spin electrons can be derived within secondorder perturbation theory [33], which results in

$$
\tilde{U} \simeq\left(U^{\prime}-J\right)-\left[2 U U^{\prime}+\left(U^{\prime}-J\right)^{2}+U^{\prime 2}\right] \chi_{\mathrm{loc}},
$$

with $\chi_{\mathrm{loc}}=\Delta \chi_{\mathrm{loc}}$ in the weak-coupling approximation [31]. If the second term on the right-hand side dominates the first-order term due to a large $\chi_{\mathrm{loc}}$, the interaction becomes attractive.

A reduction of the interaction energy below $U-3 J$ is not possible if the number of electrons is constrained to two per site, on average. On the other hand, the electrons do not have to occupy the same site when $n \leq 1$, and the superconducting state is never realized in this case.

Next, we discuss the temperature-filling phase diagram shown for $U=0.75$ in Fig. 3(a). With hole doping from half filling $(n=3)$, the AFM transition temperature decreases and becomes zero at $n \simeq 2.3$, which is close to the spin-freezing line. By further doping with holes, we
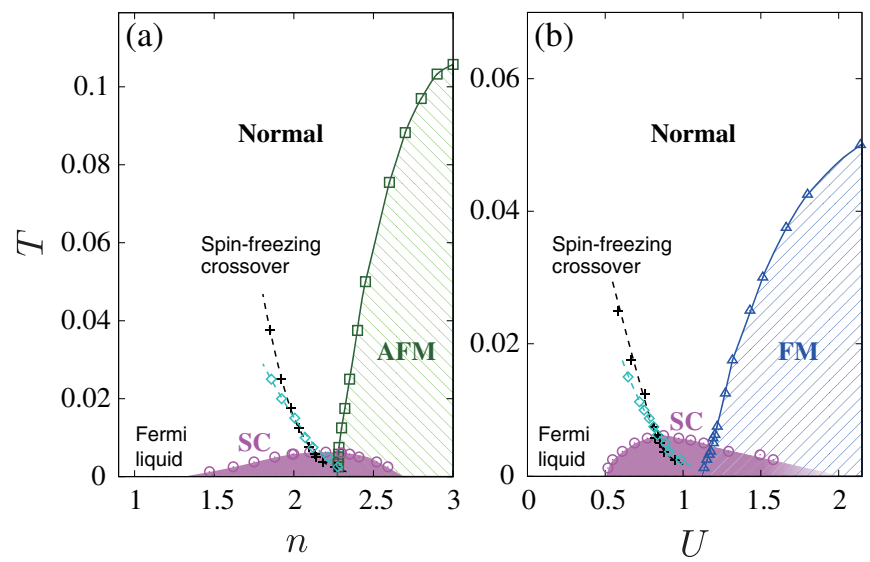

FIG. 3 (color online). (a) Temperature-filling phase diagram at $U=0.75$. (b) Temperature-interaction phase diagram at $n=2$. The black dashed line indicates the spin-freezing crossover in the system with suppressed long-range order. The light blue diamonds show the minimum of the inverse pairing susceptibility. 
find the spin-triplet SC phase with a dome-shaped $T_{c}$. If we fix the filling to $n=2$ and change $U$, we obtain the phase diagram shown in Fig. 3(b). With decreasing $U$ (increasing pressure, experimentally), the FM order is destroyed and again a SC dome appears next to the magnetic region. The light blue diamonds in Fig. 3 show the points where the inverse pairing susceptibility reaches its minimum as indicated by the arrow in Fig. 2(a). Above $T_{c}$, this corresponds to the maximal pairing instability, while below $T_{c}$ this minimum approximately locates the maximum order parameter [31]. The close resemblance to the spinfreezing crossover line shows the relevance of spin freezing for the present superconductivity.

SC domes are usually understood as a manifestation of fluctuations associated with magnetic quantum critical points. However, the superconductivity revealed in this Letter is induced by local magnetic fluctuations in the spin-freezing crossover regime. Nevertheless, the SC order naturally appears in the vicinity of a magnetic phase, since the strengthening of the magnetic moments deeper inside the spin-frozen regime causes magnetic ordering. Furthermore, the normal state above the SC dome is a non-Fermi liquid whose properties are influenced by the spin-freezing crossover [17].

Thus far we have shown results for the system with Ising anisotropy. We now clarify how the superconductivity is affected by the spin-flip term in the model with $\alpha \neq 0$. Figure 4 shows $T_{c}$ for $U=0.875$ and filling $n=2$. As $\alpha$ is increased from 0 , the transition temperature decreases and drops below the lowest accessible temperature at $\alpha=1$. The destabilization of the electron pairs by the spin-flip term can be intuitively understood by looking at Fig. 1(a). Since the spin-flip term exchanges $\uparrow$ and $\downarrow$ spin electrons residing in different orbitals, the configuration shown in the middle panel is favored. As a result, the probability for the equal-spin state (right-hand panel) decreases compared to the spin-anisotropic case. Near $\alpha=1$, the fluctuations among the three degenerate spin-triplet states further

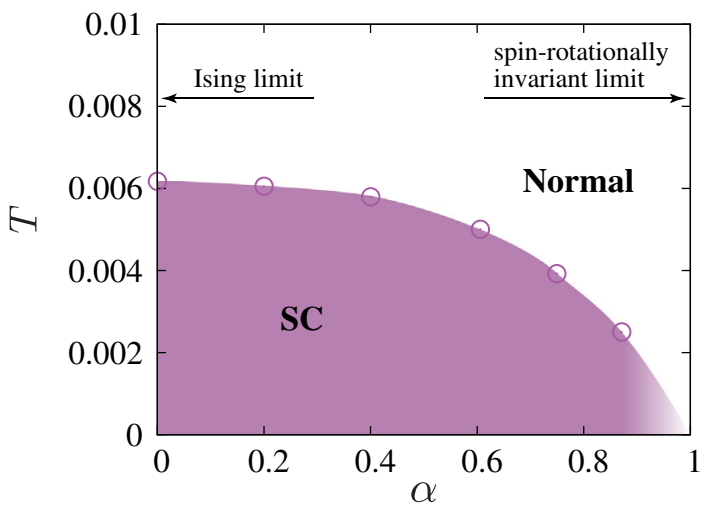

FIG. 4 (color online). Dependence of the superconducting transition temperature on the spin anisotropy $\alpha$ for $U=0.875$ and $n=2$. destabilize the equal-spin pairing. In fact, a previous study of a single-orbital model based on the Eliashberg theory [36] demonstrated the importance of longitudinal fluctuations for pairing, which is consistent with our results.

Finally, let us comment on the potential implications of these findings for unconventional multiband superconductors. Because an Ising-type spin freezing is underlying the fluctuating-moment-induced spin-triplet superconductivity, it may be expected to occur in electron systems with strong spin-orbit coupling. Promising candidates are the uraniumbased superconductors $\mathrm{UGe}_{2}$ [5], URhGe [6], and UCoGe [7], which exhibit a SC phase bordering a FM phase. In these compounds a strong Ising spin anisotropy is observed: the magnetization along the easy axis is several times larger than along the hard axis [8]. Futhermore, Ising-type spin fluctuations are important for the superconductivity, since a magnetic field along the magnetic moment destabilizes the pairing, while it is much more robust against fields perpendicular to the moment [8]. The $5 f$ electrons in the $\mathrm{U}$ ions, which play a central role in the low-temperature behavior, have a relatively itinerant nature and are strongly correlated. Although a realistic description should involve an Anderson lattice, the local interaction is of the SlaterKanamori type, and, hence, we expect the same spin-triplet SC state. We thus believe that our mechanism could be realized in these uranium-based superconductors.

$\mathrm{Sr}_{2} \mathrm{RuO}_{4}$ is another candidate compound that might exhibit a fluctuating-moment-induced superconductivity. Here, the spin-orbit coupling is nearly $100 \mathrm{meV}$ [1], and as shown in Fig. 4 the spin anisotropy need not be very large to realize a SC state at low temperatures. Also, the estimated $U \simeq 0.8$ [18] and the filling $n=4$ (same as $n=2$ due to particle-hole symmetry) place this material in the parameter regime where the SC state is found near a FM phase [Figures 2(b) and 2(c)]. A related compound, $\mathrm{SrRuO}_{3}$, with a larger $U$, becomes a ferromagnet [16] and exhibits the non-Fermi-liquid behavior associated with spin freezing in the high-temperature phase $[37,38]$.

In the iron pnictides, the Coulomb interactions and fillings on the Fe sites can also be close to the spin-freezing crossover values [20,21], and for LiFeAs, in particular, the experimental signatures fully support this interpretation [39]. On the other hand, the $3 d$ electrons have weak spin-orbit coupling, and hence a small spin anisotropy. Thus, for iron pnictides (including $\mathrm{LiFeAs}$ ), one can expect other types of pairing driven by, e.g., Fermi-surface nesting mechanisms to dominate.

For an experimental detection of the present superconductivity, it is necessary to measure both the spin and spatial parts of the pairing state. The spin part can be determined by measuring the magnetic susceptibility, e.g., with NMR. If it is identified as spin-triplet, one still has to distinguish between $p$-wave pairing and the proposed $s$-wave interorbital pairing. The difference lies in the presence (absence) of a node in the gap function which 
will be reflected in a power-law (exponential) temperature dependence of physical quantities. Our work provides a general guiding principle in the search for new unconventional multiband superconductors, namely, the combination of emerging local moments in the spin-freezing crossover regime and spin anisotropy in heavy elements.

We thank Ch. Bernhard, A. Koga, A. Millis, and M. Sigrist for helpful discussions. S. H. acknowledges financial support from JSPS KAKENHI Grant No. 13J07701 and P. W. acknowledges support from FP7 ERC starting grant No. 278023. The authors benefited from the Japan-Swiss Young Researcher Exchange Program 2014 coordinated by JSPS and SERI. The numerical calculations have been performed on the BEO04 cluster at the University of Fribourg and the supercomputer at ISSP (University of Tokyo).

[1] Y. Maeno, S. Kittaka, T. Nomura, S. Yonezawa, and K. Ishida, J. Phys. Soc. Jpn. 81, 011009 (2012).

[2] X. Dai, Z. Fang, Y. Zhou, and F.-C. Zhang, Phys. Rev. Lett. 101, 057008 (2008).

[3] S.-H. Baek, L. Harnagea, S. Wurmehl, B. Büchner, and H.-J. Grafe, J. Phys. Condens. Matter 25, 162204 (2013).

[4] J. Brand, A. Stunault, S. Wurmehl, L. Harnagea, B. Büchner, M. Meven, and M. Braden, Phys. Rev. B 89, 045141 (2014).

[5] S. S. Saxena, P. Agarwal, K. Ahilan, F. M. Grosche, R. K. W. Haselwimmer, M. J. Steiner, E. Pugh, I. R. Walker, S. R. Julian, P. Monthoux, G. G. Lonzarich, A. Huxley, I. Sheikin, D. Braithwaite, and J. Flouquet, Nature (London) 406, 587 (2000).

[6] D. Aoki, A. Huxley, E. Ressouche, D. Braithwaite, J. Flouquet, J.-P. Brison, E. Lhotel, and C. Paulsen, Nature (London) 413, 613 (2001).

[7] N. T. Huy, A. Gasparini, D. E. de Nijs, Y. Huang, J. C. P. Klaasse, T. Gortenmulder, A. de Visser, A. Hamann, T. Görlach, and H. v. Löhneysen, Phys. Rev. Lett. 99, 067006 (2007).

[8] D. Aoki and J. Flouquet, J. Phys. Soc. Jpn. 81, 011003 (2012).

[9] A. Klejnberg and J. Spalek, J. Phys. Condens. Matter 11, 6553 (1999).

[10] J. Spalek, Phys. Rev. B 63, 104513 (2001).

[11] J. E. Han, Phys. Rev. B 70, 054513 (2004).

[12] S. Sakai, R. Arita, and H. Aoki, Phys. Rev. B 70, 172504 (2004).

[13] K. Kubo, Phys. Rev. B 75, 224509 (2007).

[14] M. Zegrodnik, J. Spalek, and J. Bünemann, New J. Phys. 15, 073050 (2013).

[15] M. Zegrodnik and J. Spalek, New J. Phys. 16, 033001 (2014).
[16] A. Georges, L. d. Medici, and J. Mravlje, Annu. Rev. Condens. Matter Phys. 4, 137 (2013).

[17] P. Werner, E. Gull, M. Troyer, and A. J. Millis, Phys. Rev. Lett. 101, 166405 (2008).

[18] L. de Medici, J. Mravlje, and A. Georges, Phys. Rev. Lett. 107, 256401 (2011).

[19] K. Haule and G. Kotliar, New J. Phys. 11, 025021 (2009).

[20] A. Liebsch and H. Ishida, Phys. Rev. B 82, 155106 (2010).

[21] P. Werner, M. Casula, T. Miyake, F. Aryasetiawan, A. J. Millis, and S. Biermann, Nat. Phys. 8, 331 (2012).

[22] Z. P. Yin, K. Haule, and G. Kotliar, Phys. Rev. B 86, 195141 (2012).

[23] A. Toschi, R. Arita, P. Hansmann, G. Sangiovanni, and K. Held, Phys. Rev. B 86, 064411 (2012).

[24] M. Liu, L. W. Harriger, H. Luo, M. Wang, R. A. Ewings, T. Guidi, H. Park, K. Haule, G. Kotliar, S. M. Hayden, and P. Dai, Nat. Phys. 8, 376 (2012).

[25] A. H. Nevidomskyy and P. Coleman, Phys. Rev. Lett. 103, 147205 (2009).

[26] Although an explicit treatment of the spin-orbit coupling is necessary for a quantitative analysis, both approaches generate an anisotropy in spin space and are expected to yield similar results.

[27] A. Georges, G. Kotliar, W. Krauth, and M. J. Rozenberg, Rev. Mod. Phys. 68, 13 (1996).

[28] P. Werner and A. J. Millis, Phys. Rev. B 74, 155107 (2006).

[29] E. Gull, A. J. Millis, A. I. Lichtenstein, A. N. Rubtsov, M. Troyer, and P. Werner, Rev. Mod. Phys. 83, 349 (2011).

[30] H. Hafermann, K. R. Patton, and P. Werner, Phys. Rev. B 85, 205106 (2012).

[31] See Supplemental Material at http://link.aps.org/ supplemental/10.1103/PhysRevLett.115.247001 for origin of effective attraction, details of the computational scheme and some additional numerical data.

[32] C.-K. Chan, P. Werner, and A. J. Millis, Phys. Rev. B 80, 235114 (2009).

[33] K. Inaba and S. I. Suga, Phys. Rev. Lett. 108, 255301 (2012).

[34] Y. Okanami, N. Takemori, and A. Koga, Phys. Rev. A 89, 053622 (2014).

[35] A. Koga and P. Werner, Phys. Rev. B 91, 085108 (2015).

[36] P. Monthoux and G. G. Lonzarich, Phys. Rev. B 59, 14598 (1999).

[37] P. Kostic, Y. Okada, N. C. Collins, Z. Schlesinger, J. W. Reiner, L. Klein, A. Kapitulnik, T. H. Geballe, and M. R. Beasley, Phys. Rev. Lett. 81, 2498 (1998).

[38] J. S. Dodge, C. P. Weber, J. Corson, J. Orenstein, Z. Schlesinger, J. W. Reiner, and M. R. Beasley, Phys. Rev. Lett. 85, 4932 (2000).

[39] J. D. Wright, M. J. Pitcher, W. Trevelyan-Thomas, T. Lancaster, P. J. Baker, F. L. Pratt, S. J. Clarke, and S. J. Blundell, Phys. Rev. B 88, 060401 (2013). 